\title{
A Formal Model of Adjudication Dialogues
}

Henry Prakken

Technical Report UU-CS-2008-031

September 2008

Department of Information and Computing Sciences

Utrecht University, Utrecht, The Netherlands

www.cs.uu.nl 
ISSN: 0924-3275

Department of Information and Computing Sciences Utrecht University

P.O. Box 80.089

3508 TB Utrecht

The Netherlands 


\title{
A Formal Model of Adjudication Dialogues
}

\author{
Henry Prakken \\ Department of Information and Computing Sciences, \\ Faculty of Science, Utrecht University \\ and \\ Centre for Law \& ICT, Faculty of Law, University of Groningen \\ The Netherlands \\ henry@cs.uu.nl
}

September 22, 2008

\begin{abstract}
This article presents a formal dialogue game for adjudication dialogues. Existing AI \& law models of legal dialogues and argumentation-theoretic models of persuasion are extended with a neutral third party, to give a more realistic account of the adjudicator's role in legal procedures. The main feature of the model is a division into a pleadings phase, where the adversaries plea their case and the adjudicator has a largely mediating role, and a decision phase, where the adjudicator decides the dispute on the basis of the claims, arguments and evidence put forward in the pleadings phase. The model allows for explicit decisions on admissibility of evidence and burden of proof by the adjudicator in the pleadings phase. Adjudication is modelled as putting forward arguments, in particular undercutting and priority arguments, in the decision phase. The model reconciles logical aspects of burden of proof induced by the defeasible nature of arguments with dialogical aspects of burden of proof as something that can be allocated by explicit decisions on legal grounds.
\end{abstract}

\section{Introduction}

This article ${ }^{1}$ studies the formal modelling of the role of third parties in procedures for dispute resolution. The procedural aspects of legal reasoning have been a main research topic in AI \& Law since researchers started to realise that legal reasoning is bound not only by the rules of logic and rational inference but also by those of fair and effective procedure. This 'procedural turn' was initiated by two papers, Gordon (1993) and Hage et al. (1994), and further pursued in e.g. (Bench-Capon; 1998; Prakken and Gordon; 1998; Lodder; 1999; Hage; 2000; Vreeswijk; 2000; Leenes; 2001; Prakken; 2001b); see also Brewka (2001). The main focus of this area is the integration of logical models of legal reasoning (especially those using tools from nonmonotonic logic) with dialogue game models of argumentation. The resulting models regulate the use of argumentative speech acts, such as making, disputing and conceding claims and putting forward argument and counterarguments, and they define the outcome of a dispute in terms of the effects of these speech acts on the 'information state' of the dispute. The main guiding idea is that of procedural justice, according to which the quality of a legal decision not only depends on its content but also on how it was reached. This is partly inspired by the analogous idea of procedural rationality, defended by e.g. Toulmin (1958); Rescher (1977) and Loui (1998) (who interestingly were in turn inspired by the analogy with legal procedures).

Although all this work has been very valuable, further research is needed. Most AI \& Law work so far has concentrated on two-party dialogues between two adversaries. If the judge's role is

${ }^{1}$ This article extends, revises and simplifies Prakken (2001b). 
modelled at all, it is limited to some very simple activities, such as regulating turntaking (BenchCapon; 1998; Bench-Capon et al.; 2000) or determining the truth of the parties' claims by simply saying "true" or "false" (Hage et al.; 1994; Brewka; 2001). Yet in actual legal procedures judges have a much more elaborate role. The main aim of the present paper is to show how procedural models of legal argument can give more realistic accounts of the judge's role in legal disputes. I will focus in particular on aspects that are directly relevant for the outcome of a dispute, viz. rulings on burden of proof and admissibility of evidence, and the adjudication of the conflict in the judge's final decision. Thus I hope to clarify the relation between the logical and procedural aspects of legal reasoning.

More generally, this paper will contribute to the study of dialogue in argumentation theory. So far most studies of the dialogical aspects of legal reasoning have, either explicitly or implicitly, applied the model of so-called persuasion dialogue as developed in argumentation theory (Mackenzie; 1979; Walton and Krabbe; 1995; Prakken; 2006). In persuasion dialogues two self-interested parties aim to persuade each other that they are right and the other is wrong. Although this is clearly what happens between a plaintiff and defendant in a civil case and between prosecutor and accused in a criminal case, the persuasion model leaves no room for an adjudicator. In persuasion as modelled in argumentation theory the disagreeing parties are in full control of the outcome: if they do not want to admit that they are wrong, they cannot be forced to do so. In legal procedures, by contrast, the outcome ultimately depends on the adjudicator's decision, so in legal procedures the disagreeing parties should not persuade each other but the adjudicator. In other words, legal procedure does not fully fit the model of persuasion dialogue. Accordingly, one aim of this paper is to add a model of so-called adjudication dialogues to the study of formal dialectics in argumentation theory.

This paper is organised as follows. In Section 2 the characteristics of adjudication processes will be described in more detail, after which in section 3 the formal tools used in this paper will be introduced, viz. formal dialectics, logics for defeasible argumentation and my own framework for persuasion dialogues. Section 4, which forms the heart of this paper, extends and instantiates my persuasion framework to a three-player dialogue game for adjudication. This game is illustrated with some examples in Section 5 and more generally discussed in the concluding Section 6 .

\section{Characteristics of adjudication}

In this section the characteristics of adjudication procedures will be discussed as far as they are relevant for present purposes.

\subsection{Overview}

A typical adjudication process takes part between two adversaries who have a conflict of opinion and a neutral third party who moderates and adjudicates the conflict. The adversaries and third party will below be called plaintiff, defendant and adjudicator, respectively. Typically, a process consists of two parts, a pleadings phase in which the adversaries plea their case before the adjudicator and respond to each other, and a decision phase in which the adjudicator (whether judge or jury) decides the conflict. In the pleadings phase the adversaries make, dispute, concede and retract claims, and provide arguments for their claims or against arguments of the other adversary. The adjudicator can have various roles during the pleadings phase, such as monitoring whether the adversaries obey the procedural rules, defining the scope of the dispute by deciding what may and may not be taken into account (such as whether evidence is admissible), allocating the burden of proof and regulating turntaking and termination.

Burden of proof is one of the central notions of legal procedure. In the literature it has been argued that the burden of proof can be allocated by formalising legal rules with the proper knowledge representation techniques from nonmonotonic logic; see e.g. Sartor (1995). Although this approach works under certain assumptions, it fails to capture that in legal procedure the allocation of the burden of proof is ultimately a matter of decision by the adjudicator, and therefore 
cannot be determined by logical form alone. Any full model of reasoning under burden of proof should leave room for such decisions, and this is what I shall do, by incorporating a special speech act for allocations of the burden of proof.

Moreover, any full model of reasoning under burden of proof should take into account the well-known distinction between the burden of production and the burden of persuasion; see e.g. Strong (1992, pp. 425-6). In legal terms the burden of production specifies which party has to offer evidence on an issue at different points in a proceeding while the burden of persuasion specifies which party loses on an issue if the evidence is balanced. In the dialogue model presented in this paper the burden of production is relevant when a statement is disputed: it then specifies whether the party who made the statement must support it with an argument or whether the party who disputed it must provide an argument why it does not hold. Accordingly, in the present model the burden of production is to be allocated in the pleadings phase by means of a special speech act. The burden of persuasion, on the other hand, becomes relevant in the decision phase, when the adjudicator assesses the arguments presented by the parties. This assessment involves two aspects: whether the argument is strong enough to support the claim in the absence of counterarguments (internal validity) and whether it survives the competition with its counterarguments (dialectical validity). In the present model the adjudicator verifies the burden of persuasion in the decision phase by moving arguments, including priority arguments.

Of course, adjudication procedures can vary considerably. Turntaking and termination can be regulated in many different ways, retracting claims can be allowed or not, the adjudicator can be more or less free in assessing the evidence, and so on. Also, procedures can differ on whether the adversaries are allowed to make statements pertaining to admissibility of evidence, procedural correctness or burden of proof. The purpose of this paper is to model a fairly typical but not too complicated procedure and to focus in particular on rationality aspects of procedures instead of on their contingent legal aspects. This procedure will now be illustrated with an example.

\subsection{A motivating example}

Our formal model of adjudication should capture dialogues like the following one (which, although imaginary, is in some elements based on Dutch civil law). It contains an initial claim, decisions about the burden of production and admissibility of evidence, arguments, counterarguments and a priority argument. References to legal rules will for convenience be abbreviated as indexed letters $r$.

- Plaintiff: I claim that defendant owes me 500 euro.

- Defendant: I dispute plaintiff's claim.

- Plaintiff: Defendant owes me 500 euro by $r_{1}$ since we concluded a valid sales contract, I delivered but defendant did not pay.

- Defendant: I concede that plaintiff delivered and I did not pay, but I dispute that we have valid contract.

- Plaintiff: We have a valid contract by $r_{2}$ since this document is a contract signed by us.

- Defendant: I dispute that this is my signature.

- Plaintiff: Why?

- Judge: By $r_{3}$ the party who invokes a signature under a document which is not an avidavit has the burden to prove that it is authentic when this is disputed, so plaintiff must prove that this is defendant's signature.

- Plaintiff: This is defendant's signature since it looks just like these three signatures of which we know they are defendant's.

- Defendant: But it does not look like this signature, which is also mine. Besides, another reason why we have no contract is that I was insane when I agreed so $r_{4}$ applies, which makes Section $r_{2}$ inapplicable.

Plaintiff: I dispute that you were insane.

Defendant: My insanity is proven by this court's document, which declares me insane.

- Plaintiff: I dispute that this is a court's document.

- Judge: Plaintiff, since the document looks like a court's document, i.e., like an avidavit, by $r_{5}$ 
the burden is on you to prove that it is not.

- Plaintiff: This lab report proves that the document is forged.

Judge: This report is inadmissible as evidence by $r_{6}$ since I received it after the written pleadings phase.

Plaintiff: Nevermind, even if defendant was insane, this could not be known to me during the negotiations, so $r_{4}$ does not apply by $r_{7}$.

Defendant: Why could my insanity not be known to you?

Plaintiff: Since you looked normal all the time.

Judge (deciding the dispute): I am convinced by plaintiff's evidence that defendant's signature under the contract is authentic. Yet I cannot grant plaintiff's claim since the fact that defendant looked normal during the negotiations is insufficient to conclude that defendant's insanity could not be known to plaintiff: he might have known if he had checked the court's register. Therefore I deny plaintiff his claim.

It is beyond the scope of this paper to give a precise account of all natural-language aspects of such dialogues. To focus on the essence, the formal model will largely abstract from the formalisation of the arguments, counterarguments and priority arguments. This is not a serious limitation, since it is by now well-known in the literature on nonmonotonic logic how these things can be formalised (see e.g. Prakken and Sartor (2002) for an overview). Furthermore, it is not my aim to account for the fact that often elements of arguments are left implicit. I believe that this issue is far from trivial and therefore deserves a separate study. Accordingly, I will in this paper only allow fully explicit arguments.

\section{$2.3 \quad$ Aspects to be modelled}

I now list in more detail the activities to be modelled.

The adversaries' acts:

- Stating, disputing, retracting and conceding claims.

- Stating arguments and counterarguments.

The adjudicator's acts:

- Deciding about procedural correctness of moves (which includes admissibility of evidence).

- Deciding about the burden of production.

- Deciding about termination of a dispute.

- Deciding whether a party has met its burden of persuasion. This involves deciding about the following issues:

- whether an argument is able to support its conclusion even in the absence of counterarguments (internal validity);

- whether the argument survives competition with its counterarguments (dialectical validity).

Some of these acts will be modelled with special speech acts (viz. ruling moves legally inadmissible, allocating the burden of production and terminating the dialogue), while the internal and dialectical assessment of arguments will be modelled as specific ways to move arguments.

\section{An overview of the formal tools}

In this section the formal tools used in this paper will be introduced. 


\subsection{Formal dialectics and dialogue games}

Procedural AI \& Law models have largely been based on a branch of argumentation theory and philosophical logic called 'formal dialectics' (Hamblin; 1971; Mackenzie; 1979; Walton and Krabbe; 1995), which formalises rules for dialogues involving argumentation, in particular persuasion dialogues. The main aim of dialogue systems is to define conventions for coherent discourse, where an utterance in a dialogue is regarded as coherent if it contributes to the goal of the dialogue (Carlson; 1983). The goal of adjudication dialogues can be described as fair and effective dispute resolution.

Most work on formal dialectics takes a game-theoretic approach to dialogues, where speech acts are viewed as moves in a game and rules for when these moves are allowed are formulated as rules of the game. More specifically, formal dialogue games have a topic language $L_{t}$ with a logic $\mathcal{L}$, and a communication language $L_{c}$ with a protocol $P$. The protocol specifies the allowed moves at each point in a dialogue. A dialogue system also has effect rules, which specify the effects of utterances on the participants' commitments, and termination and outcome rules.

AI \& Law models have extended dialogue games for persuasion with the possibility of counterargument. While in the systems of formal dialectics the only way to challenge an argument is by disputing its premises, in the AI \& Law models a party can challenge an argument even if $\mathrm{s} /$ he accepts all premises, viz. by stating a counterargument. In other words, while in the argumentation-theoretic models the underlying logic is deductive, in the AI \& Law systems it is defeasible.

A formal underpinning for the latter is provided by the study of argument games in artificial intelligence, to which I now turn.

\subsection{Logics for defeasible argumentation}

The idea that legal reasoning is defeasible is generally accepted in AI \& Law and is increasingly accepted in legal philosophy (Peczenik; 1996; Hage; 1997; Sartor; 2005). Here I will simply take this idea for granted. In formalising it, I will take an argumentation approach, since the dialectical flavour of this approach fits well with protocols for dialogue and procedure. Providing grounds and evidence will be modelled as constructing arguments for a claim, and attacking grounds and providing counterevidence will be modelled as constructing counterarguments. Three ways of attacking arguments will be assumed, viz. attacking a premise with an argument for its negation (premise attack), attacking the conclusion with an argument for its negation (rebutting attack) and attacking the support relation between the premises and the conclusion (undercutting attack). The latter two attacks can also be targeted at intermediate conclusions or inference steps of an argument. Finally, adjudication will also be modelled as constructing arguments, typically as constructing priority arguments, which are arguments that break ties between conflicting arguments.

Formal systems for defeasible argumentation, or argumentation systems for short, have been developed in the past twenty years in artificial intelligence as models of common-sense reasoning. (See Prakken and Vreeswijk (2002) for an overview.) These systems formalise defeasible reasoning as the construction and comparison of arguments for and against certain conclusions. They define how arguments can be constructed from a given body of information, how such arguments can be attacked by counterarguments, and how conflicting arguments can be compared in terms of given criteria for comparison. To all arguments that can be constructed a so-called dialectical status is then assigned. Typically this status is defined in terms of three classes: the 'winning' or justified arguments, the 'losing' or overruled arguments, and the 'ties', i.e., the defensible arguments, which are involved in an irresolvable conflict. In this paper I will especially be interested in determining whether an argument is justified, since the main aim of an adjudication procedure is to decide whether a justified argument exists for a claim.

Argumentation logics can be defined with fixpoint definitions but also in the dialectical form of argument games (cf. Loui; 1998). Such games model defeasible reasoning as a dispute between a proponent and opponent of a statement. In this paper the argument game of Prakken and Sartor (1997) as modified by Prakken (2001a) will be used. The game of Prakken and Sartor (1997) is 
defined as follows. Let ' $A$ defeats $B$ ' stand for ' $A$ attacks $B$ and is not worse than $B$ ' and note that two conflicting arguments defeat each other if they are equally strong or their relative strength is unknown. In the game proponent starts with an argument that he wants to prove justified and then each player must either defeat the other player's previous argument or move a priority argument that stops the previous argument from defeating its target. Moreover, proponent is not allowed to repeat an argument in attack on the same argument, since if opponent had a reply the first time, she will also have a reply the next time. A player wins if the other player has run out of moves. The initial argument is provably justified if the proponent has a winning strategy in this game.

Note that under these rules the proponent will win only if his arguments strictly defeat opponent's counterarguments, that is, only if he moves arguments that are not defeated by their target. In other words, the proponent loses when the evidence is balanced, so he in fact has the burden of persuasion for all his claims. This does not agree with the fact that in the law the burden of persuasion can be distributed over the parties. Accordingly, in Prakken (2001a) I modified the argument game by making the proponent and opponent roles relative to statements. Each player (plaintiff or defendant) now has proponent role for the statements for which they have the burden of persuasion, while they have opponent role for statements for which the other player has the burden of persuasion. Assignments of the burden of persuasion are simply modelled as additional input to the logic. It is this version of the game that I will use in the present paper.

Clearly, the idea of argument games fits well with formal dialectics. However, for present purposes they also have an important limitation: they are static in that, being proof theories for logics, they operate on a fixed body of information from which conclusions are drawn. By contrast, in argumentation dialogues the information on which the outcome of a dialogue is determined is usually created dynamically during a dialogue. For this reason I showed in Prakken (2005) how argument games can be embedded in dialogue systems for persuasion. The present task is to extend the (two-player) persuasion model of that paper to a (three-player) model of adjudication. To this end the main elements of Prakken (2005) will be summarised next.

\subsection{A framework for two-player persuasion dialogue}

In Prakken (2005) a framework for specifying two-party persuasion dialogues is presented, which is then instantiated with some example protocols. Below the main elements of the instantiation used in this paper are summarised. Readers in need of illustration may wish wish to look ahead to Section 5.2, where the example of Section 2.2 is visualised.

A main motivation of the framework is to ensure focus of dialogues while yet allowing for freedom to move alternative replies and to postpone replies. This is achieved with two main features of the framework.

The first is an explicit reply structure on the communication language. Each dialogue move except the initial one replies to one earlier move in the dialogue of the other party (its target). Thus a dialogue can be regarded in two ways: as a sequence (reflecting the order in which the moves are made) and as a tree (reflecting the reply relations between the moves). Each replying move is either an attacker or a surrender. For instance, a claim $p$ move can be attacked with a why $p$ move and surrendered with a concede $p$ move; and a why $p$ move can be attacked with an argue $A$ move where $A$ is an argument with conclusion $p$, and surrendered with a retract $p$ move. When $s$ is a surrendering and $s^{\prime}$ is an attacking reply to $s^{\prime \prime}$, we say that $s^{\prime}$ is an attacking counterpart of $s$.

The second idea is that at each stage of a dialogue, each dialogue move has a dialogical status, which is either in or out. It is recursively defined as follows, exploiting the tree structure of dialogues. A move is in if it is surrendered or else if all its attacking replies are out. (This implies that a move without replies is in). And a move is out if it has a reply that is in. (Actually, this has to be refined to allow that some premises of an argument are conceded while others are disputed; see Prakken (2005) for the details). This allows the definition of the current winner of a dialogue: a dialogue is (currently) won by the plaintiff if its initial move is in while it is (currently) won by 
the defendant otherwise. Figures 1 and 2 in Section 5.2 illustrate the reply structure of dialogues and the dialogical status of moves.

As for dialogue structure, the framework allows for all kinds of variations. The instantiation used here is very liberal in its structural aspects: essentially, both players can speak whenever they like, except that they cannot speak at the same time. Also, they may reply to any earlier move of the other player instead of having to reply to the last such move, and they may move alternative replies to the same move, possibly even in the same turn (a turn is a sequence of moves of one player). Other protocols defined in Prakken (2005) impose restrictions on these points; since dialogue structure is not our present concern, they will not be discussed here.

The framework largely abstracts from the topic language and its logic but arguments are assumed to be trees of deductive and/or defeasible inferences. Recall that in the present paper the logic of Prakken (2001a) will be assumed. To keep things simple, formal details of language and logic will be omitted.

In Prakken (2005) dialogues are between a proponent $P$ and opponent $O$ of a single dialogue topic $t \in L_{t}$. However, to allow for distributions of the burden of persuasion over the parties, in the present paper I adopt Prakken (2001a)'s distinction between dialogue parties (plaintiff $(\pi)$ and defendant $(\delta)$ ) and their dialectical roles towards particular statements (proponent and opponent). The dialectical roles are specified in a function that at each stage of a dialogue assigns a (possibly empty) set of statements to each player for which they have the burden of persuasion. Each such set is consistent and is such that if one player has the burden of persuasion for $\varphi$, no other player has the burden of persuasion for either $\varphi$ or $\neg \varphi$.

Now the protocol is based on the following ideas.

The communication language $L_{c}$ assumed in this paper is specified in Table 1. In this table, $\varphi$ is from $L_{t}$ and arguments $A$ and $B$ are well-formed arguments from $\mathcal{L}$.

\begin{tabular}{|l|l|l|}
\hline Acts & Attacks & Surrenders \\
\hline \hline claim $\varphi$ & why $\varphi$ & concede $\varphi$ \\
\hline why $\varphi$ & argue $A(\operatorname{conc}(A)=\varphi)$ & retract $\varphi$ \\
\hline argue $A$ & why $\varphi(\varphi \in \operatorname{prem}(A))$, & concede $\varphi$ \\
& argue $B$ & $(\varphi \in \operatorname{prem}(A)$ or \\
& & $\varphi=\operatorname{conc}(A))$ \\
\hline concede $\varphi$ & & \\
\hline retract $\varphi$ & & \\
\hline
\end{tabular}

Table 1: A communication language for persuasion

A protocol for $L_{c}$ is defined in terms of the notion of a dialogue, which in turn is defined with the notion of a move.

\section{Definition 3.1 (Dialogues)}

- The set $M$ of moves is defined as $\mathbb{N} \times\{\pi, \delta\} \times L_{c} \times \mathbb{N}$, where the four elements of a move $m$ are denoted by, respectively:

- id(m), the identifier of the move,

- $p l(m)$, the player of the move,

- $s(m)$, the speech act performed in the move,

$-t(m)$, the target of the move.

- The set of dialogues, denoted by $M \leq \infty$, is the set of all sequences $m_{1}, \ldots, m_{i}, \ldots$ from $M$ such that

- each $i^{\text {th }}$ element in the sequence has identifier $i$,

$-t(m)=0$ iff $m=m_{1}$ or for no $s \in L c$ it holds that $s(m)$ is a reply to $s$ in $L_{c}$; 
- If $t(m) \neq 0$ then $t(m)=i$ for some $m_{i}$ preceding $m$ in the sequence.

The set of finite dialogues, denoted by $M^{<\infty}$, is the set of all finite sequences that satisfy these conditions. For any dialogue $d=m_{1}, \ldots, m_{n}, \ldots$, the sequence $m_{1}, \ldots, m_{i}$ is denoted by $d_{i}$, where $d_{0}$ denotes the empty dialogue.

When $t(m)=i d\left(m^{\prime}\right)$ I say that $m$ replies to $m^{\prime}$ in $d$ and that $m^{\prime}$ is the target of $m$ in $d$. Abusing notation I sometimes let $t(m)$ denote a move instead of just its identifier. When $s(m)$ is an attacking (surrendering) reply to $s\left(m^{\prime}\right)$ I also say that $m$ is an attacking (surrendering) reply to $m^{\prime}$.

Protocols are in Prakken (2005) defined as follows.

Definition 3.2 (Protocols) A protocol on $M$ is a set $P \subseteq M^{<\infty}$ satisfying the condition that whenever $d$ is in $P$, so are all initial sequences that $d$ starts with.

A partial function $\operatorname{Pr}: M^{<\infty} \longrightarrow \mathcal{P}(M)$ is derived from $P$ as follows:

- $\operatorname{Pr}(d)=$ undefined whenever $d \notin P$;

- $\operatorname{Pr}(d)=\{m \mid d, m \in P\}$ otherwise.

The elements of $\operatorname{dom}(\mathrm{Pr})$ (the domain of $\mathrm{Pr}$ ) are called the legal finite dialogues. The elements of $\operatorname{Pr}(d)$ are called the moves allowed after $d$. If $d$ is a legal dialogue and $\operatorname{Pr}(d)=\emptyset$, then $d$ is said to be a terminated dialogue.

These protocol rules for move legality should not be confused with the rules of legal procedure that are checked by a judge. The protocol rules are rational rules for any adjudication dialogue while a judge only checks the contingent rules of a specific procedure, such as the rules on admissibility of evidence. See Vreeswijk (2000) for a dialogue protocol in which the protocol rules can themselves be debated within the protocol.

All protocols are further assumed to satisfy the following basic conditions for all moves $m_{i}$ and all legal finite dialogues $d$.

If $m \in \operatorname{Pr}(d)$, then:

- $R_{1}: p l(m) \in T(d) .^{2}$

- $R_{2}$ : If $t(m) \neq 0$ then $s(m)$ is a reply to $s(t(m))$ according to $L_{c}$.

- $R_{3}$ : If $m$ replies to $m^{\prime}$, then $p l(m) \neq p l\left(m^{\prime}\right)$.

- $R_{4}$ : If there is an $m^{\prime}$ in $d$ such that $t(m)=t\left(m^{\prime}\right)$ then $s(m) \neq s\left(m^{\prime}\right)$.

- $R_{5}$ : For any $m^{\prime} \in d$ that surrenders to $t(m), m$ is not an attacking counterpart of $m^{\prime}$.

Rule $R_{1}$ says that the player of a move must be to move. Rules $R_{2}-R_{4}$ formalise the idea of a dialogue as a move-reply structure that allows for alternative replies. Rule $R_{5}$ says that once a move is surrendered, it may not be attacked any more. Note that these five protocol rules only state necessary conditions for legality of moves. They can be completed in many ways with further conditions, for instance, to prevent circular dialogues (Mackenzie; 1979; Walton and Krabbe; 1995) but to focus on the essence such rules will be left undiscussed here. However, two additional rules are assumed in this paper. The first says that each dialogue begins with either a claim or an argument and the second constrains the moving on arguments.

- $R_{6}$ : If $d=d_{0}$ then $s(m)$ is of the form claim $\varphi$ or argue $A$.

- $R_{7}$ : If an argue $A$ move replies to an argue $B$ move then

${ }^{2} T(d)$ denotes the player(s) whose turn it is to move in $d$. 
1. if $A$ is moved in proponent role then the player who moves it has not already moved $A$ in the same line of dialogue; and

2. if $A$ does not defeat $B$ then $B$ replies to an argue $C$ move such that $B$ defeats $C$ and $A$ is a priority argument that stops $B$ from defeating $C$.

In rule $R_{7}$ a dialogue line is a single branch of the dialogue tree induced by the reply relations between moves. The purpose of this rule is to build the argument game of Prakken (2001a) into the protocol.

The commitment rules of a dialogue system define the effects of a move on the players' propositional commitments. For instance, a claim makes a player committed to its content and a retraction terminates such commitment. In several dialogue games commitments are an important ingredient (see e.g. Walton and Krabbe (1995)). However, in the present approach their role is largely performed by the reply structure on the communication language and the notion of dialogical status. Therefore, they will not be further discussed here.

As for turntaking it was noted above that both players can speak whenever they like, except that they cannot speak at the same time. Termination was in Definition 3.2 implicitly defined as the situation where the player(s) to move cannot make a legal move. In Section 4 this 'mathematical' definition of termination will be reconciled with the nature of adjudication.

The link with the underlying argumentation logic is made in terms of a so-called dialectical graph, which records all the arguments stepwise constructed during the dialogue, as well as their defeat relations. The idea is that during a persuasion dialogue this graph is extended by stating arguments for claims, by stating counterarguments and by extending arguments with arguments for their premises. The full definitions can be found in Prakken (2005). Figure 3 in Section 5.2 provides an illustration.

Ideally, the outcome of the dialogue and of the dialectical graph correspond in the following way. The initial move of a dialogue is in just in case the 'defended part' of the dialectical graph, that is, the set of arguments of which no premise is disputed, makes an argument for the initial claim justified. In Prakken (2005) it is proven that this holds on two conditions: that no surrenders are moved (since a player can, for instance, concede or retract a claim even if he logically does not have to) and if the dialogue is 'logically completed', i.e., if no new relevant arguments can be moved in the dialogue without stating new premises.

\section{Adapting the dialogue system to adjudication}

In this section the above framework for two-player persuasion dialogues will be extended to adjudication. A third player, the adjudicator, will be added, some new speech acts introduced and the protocol, turntaking and termination rules will be adapted.

\subsection{Overview}

I first sketch how an adjudication dialogue according to the new framework generally evolves. Each dialogue starts with the pleadings phase and does so with a claim of the plaintiff, who takes proponent role with respect to this claim. During the dispute about the claim the adversaries together implicitly build an argument graph in the manner defined above. More precisely, when a claim is disputed, the adversaries can exchange arguments and counterarguments and can dispute their premises. Sometimes, disagreements are resolved peacefully by conceding or retracting claims. The adversaries can also try to shift the burden of production on the other adversary by replying to a disputing of $\varphi$ with disputing the opposite of $\varphi$. When an adversary has ended his turn, the turn shifts to the adjudicator. The adjudicator first decides on the procedural correctness of the moves made in the previous turn. Then he can allocate the burden of production of propositions that have been disputed and finally he decides whether to terminate the pleadings phase. If the dialogue continues, then the turn shifts to the current loser at the end of the adjudicator's turn. 
In the decision phase only the adjudicator can move and until termination he can only move arguments, including counterarguments and priority arguments, to determine whether the various burdens of persuasion have been met. These burdens are assumed to have been detemined by the judge between the pleadings and decision phase and are given as input to the decision phase. (The model could be extended with the means for the judge to express her reasons for these allocations as arguments; this can be done by replacing the logic of Prakken (2001a) as the underlying logic of $L_{t}$ with the one of Prakken and Sartor (2007), in which arguments about the burden of persuasion can be expressed.) When the decision phase terminates, the winner is determined by the dialogical status of plaintiff's main claim.

\subsection{The new dialogue game}

A dialogue now takes place between three players. The set Players $=\{\pi, \delta, \alpha\}$, where $\pi$ and $\delta$ are the adversaries and $\alpha$ the adjudicator. The variable $p$ ranges over all three players, while the variable $a$ ranges over the adversaries. If $a$ is an adversary, then $\bar{a}$ denotes the other adversary. So $\bar{\pi}=\delta$ and $\bar{\delta}=\pi$.

As for the the communication language, the addition of an adjudicator requires some new speech acts. Firstly, with respect to turntaking the idea now is that after a turn of an adversary the turn shifts to the adjudicator, who must decide whether the moves made by the speaker were legally admissible and whether there is reason to make an explicit decision about the burden of production. Therefore, the end of an adversaries' turn must now be recognisable by the adjudicator so all turns must now end with a pass speech act. Secondly, the adjudicator has the power to terminate a dialogue, which he can do with a terminate speech act. These two speech acts do not reply to and cannot be replied-to by other speech acts. According to Definition 3.1 above their target therefore is the dummy value 0 .

Next, from now on the speech act why $\varphi$ can also be used in attack of a $w h y \neg \varphi$ move, to express that the speaker claims that the burden of production for the opposite of $\varphi$ is on the hearer (an idea taken from Prakken et al. (2005) and originally due to Chris Reed). More generally, the second why move can dispute the 'complement' of the first disputation. To capture this the notation $-\varphi$ is introduced, which denotes the complement of $\varphi$ : when $\varphi$ is not a negation its complement is $\neg \varphi$, otherwise, its complement deletes the negation sign. So, for instance, the complement of $p$ is $\neg p$ and the complement of $\neg p$ is $p$.

Finally, new speech acts are needed for deciding about the burden of production and about procedural correctness of a move, viz. $\operatorname{burden}(\varphi, p)$ and illegal $m$. Here burden $(\varphi, p)$ means that player $p$ has the burden of production for $\varphi$ and illegal $m$ means that move $m$ violates the rules of legal procedure. (Recall that these rules are not the same as the one of the dialogue game.) An illegal move can be made in reply to any other move while a burden $(\varphi, p)$ move can only be made in reply to a why $\varphi$ move.

The resulting communication language is displayed in Figure 2. Next, the following protocol rules are added to rules $R_{1}-R_{7}$. They distinguish two phases of a dialogue: the pleadings phase is the phase before a terminate move has been moved and the decision phase is the remaining phase.

- $R_{8}$ : A terminate, burden and illegal move may only be moved by the adjudicator. In the pleadings phase the adjudicator only moves burden, illegal, pass and terminate moves. In the decision phase the adjudicator only moves argue and terminate moves.

- $R_{9}$ : In the pleadings phase each of the adjudicator's moves replies to a move from the previous turn, except when $R_{10}$ applies.

- $R_{10}$ : If the previous turn contains a why $-\varphi$ attack $m$ on a why $\varphi$ move $m^{\prime}$ and the adjudicator does not rule $m$ illegal then he attacks either $m$ or $m^{\prime}$ with a burden move.

- $R_{11}$ : Each burden move assigns the burden of production to the speaker of the move's target.

- $R_{12}$ : A move with an illegal or burden reply may not be replied-to any more. 


\begin{tabular}{|l|l|l|}
\hline Acts & Attacks & Surrenders \\
\hline \hline claim $\varphi$ & $\begin{array}{l}\text { why } \varphi \\
\text { illegal } m\end{array}$ & concede $\varphi$ \\
\hline why $\varphi$ & $\begin{array}{l}\text { argue } A(\operatorname{conc}(A)=\varphi) \\
\text { why }-\varphi \\
\text { burden }(-\varphi, a) \\
\text { illegal } m\end{array}$ & retract $\varphi$ \\
\hline argue $A$ & $\begin{array}{l}\text { why } \varphi(\varphi \in \text { prem }(A)), \\
\text { argue } B\end{array}$ & $\begin{array}{l}\text { concede } \varphi \\
(\varphi \in \operatorname{prem}(A) \text { or } \\
\varphi=\operatorname{conc}(A))\end{array}$ \\
\hline concede $\varphi$ & illegal $m$ & \\
\hline retract $\varphi$ & illegal $m$ & \\
\hline illegal $m$ & & \\
\hline burden $(\varphi, p)$ & & \\
\hline pass & & \\
\hline terminate & & \\
\hline
\end{tabular}

Table 2: A communication language for adjudication dialogues

- $R_{13}$ : After the first terminate move no move by an adversary is legal. After a second terminate move no move is legal.

- $R_{14}$ : If the current winner cannot become the current loser at a later stage, the adjudicator can only move a terminate move.

Rule $R_{8}$ captures the differences between the pleadings and the decision phase. Rules $R_{9}$ and $R_{10}$ implement the idea that issues of move legality and burden of production are dealt with as soon as they arise. Rule $R_{11}$ ensures that the player to whom the burden of production is allocated is the current loser, so that he has to support the disputed statement with an argument. Rule $R_{12}$ captures that decisions on move legality and the burden of production are irreversible and undisputable. Rule $R_{13}$ marks the shift from the pleadings to the decision phase. Finally, $R_{14}$ in fact captures that if the adversaries have peacefully resolved the dispute, the adjudicator cannot change the outcome and has to terminate the dispute. This is so since the only way in which plaintiff, respectively, defendant can become the permanent current winner is if defendant concedes, respectively plaintiff retracts the main claim.

The new turntaking rules are as follows.

- $T_{1}$ : plaintiff starts a dispute.

- $T_{2}$ : In the pleadings phase the turn only shifts after a pass move.

- $T_{3}$ : After a pass move by an adversary the turn shifts to the adjudicator.

- $T_{4}$ : After a pass move by the adjudicator the turn shifts to the current loser.

- $T_{5}$ : In the decision phase it is always the adjudicator's turn.

Termination was in Definition 3.2 implicitly defined as the situation where the player(s) to move cannot make a legal move. However, in legal settings a dispute is often arbitrarily ended after a fixed number of turns or by the adjudicator's intervention. This is why the locution terminate was added to $L_{c}$ and two further protocol conditions were added that only the third party can move this locution and that after such a move no other move is legal.

Finally, the outcome rule says that the winner of a dispute is the current winner at termination of the decision phase. 


\subsection{The adjudicator's options in the decision phase}

Technically speaking the decision phase does not fit the framework of this paper and Prakken (2005) since it is a monologue and since the adjudicator can reply to his own moves. (This makes sense since sometimes a decision takes the form of an internal debate, such as "in general, in cases like these $p$ holds but in this case there is an exception".) However, most rules of our framework will also be assumed to hold for the decision phase. To this end the requirement of the general framework that players cannot reply to their own moves is dropped for the decision phase. Next, recall that the purpose of the decision phase is to decide the dispute. This involves deciding about a number of issues. Let us see how the new dialogue game enables the adjudicator to express these decisions.

- Deciding whether a non-disputed and non-attacked premise of an argument holds. If not, the adjudicator moves a premise-attacking counterargument, otherwise no response to the premise is needed.

- Deciding whether an argument is internally valid, that is, whether it is acceptable if not attacked by counterarguments. If it is not, then the adjudicator expresses this by moving an undercutting counterargument, otherwise the following decisions must be made.

- Deciding whether there is reason to move additional counterarguments to internally valid arguments.

- Deciding whether an argument is dialectically valid, i.e., whether it survives the competition with its internally valid counterarguments. This is typically decided by priority arguments.

Note that all these decisions can be made by either remaining silent or moving an argument.

\section{$5 \quad$ Examples}

In this section the new dialogue game will be illustrated with some examples. As for notation, the target of a move will be indicated in square brackets and argue moves will be displayed as 'conclusion since premises'. Initially, I assume that the plaintiff has the burden of persuasion for all statements made by any player; at the end of this section I will briefly consider a situation where defendant also has the burden of persuasion for some claims.

\subsection{Abstract examples}

Let us first illustrate the working of the system with a few symbolic examples.

$\pi_{1}: \operatorname{claim} p$

$\pi_{2}:$ pass

The turn shifts to the adjudicator, whose only legal moves are to rule $\pi_{1}$ illegal, to terminate the dialogue or to pass. Initial claims are hardly ever illegal and it does not make much sense to terminate a dialogue after the first move, so the adjudicator passes. Since plaintiff then trivially is the current winner since his initial moves has no replies, the turn shifts to defendant.

$\alpha_{3}:$ pass

$\delta_{4}\left[\pi_{2}\right]:$ why $p$

$\delta_{5}:$ pass

The adjudicator must now decide about legality of $\delta_{4}$. He implicitly rules it legal by moving no illegal $\delta_{4}$ reply. Next the adjudicator must decide about the burden of production for $p$. Note that at this point $\pi_{1}$ is out so plaintiff is the current loser, which means that 'by default' the 
burden of production is on him. The adjudicator sees no reason to place it on defendant nor to terminate the dialogue, so he passes. Note that if defendant had not disputed but conceded $p$, move $\pi_{1}$ would have been made in for ever, so if the adjudicator had ruled the concession legal, he would have been forced to terminate the dialogue by rule $R_{14}$.

$\alpha_{6}: p a s s$

Plaintiff now has the choice to accept the default burden of production and defend $p$ with an argument or to try to place the burden on defendant. Let us assume he accepts the default burden of production.

$\pi_{7}\left[\delta_{4}\right]: p$ since $q$

$\pi_{8}$ : pass

$\alpha_{9}:$ pass

Now an interesting situation has arisen. Since at this point defendant is the current loser, plaintiff has with $\pi_{7}$ managed to shift the burden of proof on her in a certain sense. To avoid losing in the end, defendant must do something. She could dispute $\pi_{7}$ 's premise but she could also move a counterargument against $\pi_{7}$ 's argument. In both cases the defendant becomes the current winner so she has managed to shift this new sense of burden of proof back to plaintiff. This type of proof burden is sometimes called the tactical burden of proof (see Prakken and Sartor (2006) for a more detailed discussion and references to the jurisprudential literature). In the context of a legal proceeding it is the burden to do something that makes oneself the likely winner of the proceeding if the resulting situation were the final situation on the basis of which the proceeding is decided. In terms of the present dialogue game it is the burden to do something that makes oneself the current winner. Note that the tactical burden of proof is, unlike the burdens of production and persuasion, not a matter of law or judicial decision but is induced by the dialogue rules and the (nonmonotonic) logic.

Suppose defendant decides to dispute $\pi_{7}$ 's premise.

$\delta_{10}\left[\pi_{7}\right]:$ why $q$

$\delta_{11}$ : pass

Let us now examine the two ways in which the burden of production can become the subject of explicit dispute. In our dialogue game the burden of production concerning a statement is by default on the one who made that statement in that if the other player disputes it and the judge remains silent, the first player is the current loser. In our example this is the situation after $\alpha_{6}$. There are two ways in which the burden of production for a statement (in our case $q$ ) can be placed on the one who disputed it. The first is that the adjudicator directly after the why $p$ move decides that the burden to prove $\neg q$ is on defendant.

$\alpha_{12}\left[\delta_{10}\right]: \operatorname{burden}(\neg q, \delta)$

$\alpha_{13}$ : pass

Since $\alpha_{13}$ has made $\delta_{10}$ out, defendant is the current loser. To become the current winner she can move an undercutting or rebutting counterargument to $\pi_{7}$ or she can fulfill the burden of production for $\neg q$ by moving a premise attack on $\pi_{7}$ with an argument for $\neg q$.

Assume next that the adjudicator instead wants to wait and see if plaintiff accepts the default burden of production for $q$.

$\alpha_{12}^{\prime}:$ pass

Suppose plaintiff instead tries to place the burden of production on defendant. 
$\pi_{13}\left[\delta_{10}\right]:$ why $\neg q$

$\pi_{14}:$ pass

Then the adjudicator can still decide to shift the burden to defendant with

$\alpha_{15}\left[\delta_{10}\right]: \operatorname{burden}(\neg q, \delta)$

after which defendant has the same options as after $\alpha_{13}$.

This example illustrates that if the burden of production for a premise is placed on the party that disputed it, that party can only fulfill it by moving a premise-attacking counterargument.

\subsection{The motivating example formalised}

Let us next formalise the example of Section 2.2. For simplicity, the pass moves are left implicit.

$\pi_{1}:$ claim owe 500

$\delta_{2}\left[\pi_{1}\right]$ : why owe 500

$\pi_{3}\left[\delta_{2}\right]$ : owe 500 since contract and delivered and $\neg$ paid and $r_{1}$

$\delta_{4}\left[\pi_{3}\right]$ : concede delivered

$\delta_{5}\left[\pi_{3}\right]$ : concede $\neg$ paid

$\delta_{6}\left[\pi_{3}\right]:$ why contract

Again the turn shifts to plaintiff. Since he knows the general rule of Dutch civil procedure that the one who claims that a legal relation exists has to prove it, he again accepts the default burden of production.

$\pi_{7}\left[\delta_{6}\right]$ : contract since document and signed and $r_{2}$

$\delta_{8}\left[\pi_{7}\right]$ : why signed

Now plaintiff tries to place the burden of producing evidence that there is something wrong with the signatures on defendant.

$\pi_{9}\left[\delta_{8}\right]:$ why $\neg$ signed

The adjudicator implicitly rules $\pi_{9}$ legal by moving no illegal reply and is then forced by rule $R_{10}$ to decide about the burden of production with respect to 'signed by us', since the burden is now in dispute. If he wants to assign it to defendant then he must reply to $\delta_{8}$ with a burden $(\neg$ signed by us, $\delta$ ) move. But in agreement with Dutch civil procedure he instead allocates the burden to plaintiff, which makes plaintiff the current loser so the turn shifts back to him.

$\alpha_{10}\left[\pi_{9}\right]$ : burden (signed, $\pi$ )

Note that the present formalism does not allow the adjudicator to express his reasons why the burden of production is on plaintiff. Such a possibility could be added but for simplicity I will not pursue this here.

$\pi_{11}\left[\delta_{8}\right]:$ signed since similar-to-3

Plaintiff has fulfilled his burden of production so the tactical burden of proof now automatically shifts to defendant, who has to provide counterevidence in the form of a rebutting counterargument.

$\delta_{12}\left[\pi_{11}\right]: \neg$ signed since $\neg$ similar-to-1 
Afer this rebuttal defendant also plays out her second attack on plaintiff's main argument, backtracking to $\pi_{7}$ :

$\delta_{13}\left[\pi_{7}\right]: \neg r_{2}$ since insane and $r_{4}$

$\pi_{14}\left[\delta_{13}\right]:$ why insane

$\delta_{15}\left[\pi_{14}\right]$ : insane since court's doc and declares insane

$\pi_{16}\left[\delta_{15}\right]$ : why court's doc

At this point the adjudicator allocates the burden of production to plaintiff, since by Dutch law something that looks like an avidavit is presumed to be an avidavit. This presumption can only be overturned by counterevidence.

$\alpha_{17}\left[\pi_{16}\right]: \operatorname{burden}(\neg$ court's doc, $\pi)$

Plaintiff provides counterevidence by stating a premise attack on $\delta_{15}$.

$\pi_{18}\left[\delta_{15}\right]: \neg$ court's doc since lab report and says that forged

The adjudicator now rules the lab report inadmissible, after which the turn shifts back to plaintiff.

$\alpha_{19}\left[\pi_{18}\right]:$ illegal $\pi_{18}$

Plaintiff now backtracks to $\delta_{13}$, arguing that there is an exception to the exception.

$\pi_{20}\left[\delta_{13}\right]: \neg r_{4}$ since could not know and $r_{7}$

$\delta_{21}\left[\pi_{20}\right]$ : why could not know

$\pi_{22}\left[\delta_{21}\right]$ : could not know since looked normal

$\alpha_{23}$ : terminate

The adjudicator terminates the pleadings phase of the dispute and enters the decision phase, in which he must decide about the internal and dialectical validity of the various arguments. Recall that at this point it is simply assumed that the burdens of persuasion are known (the judge allocates these burdens between the pleadings and decision phase by applying the relevant procedural rules).

The adjudicator first rules that plaintiff has met his burden of persuasion for 'signed by us' by moving a priority argument against $\delta_{12}$.

$\alpha_{24}\left[\delta_{12}\right]: \pi_{11}>\delta_{12}$ since convinced by $\pi_{11}$

This priority argument stops $\delta_{12}$ 's argument from defeating $\pi_{11}$ 's argument, so the latter has been ruled dialectically valid. Next the adjudicator rules plaintiff's argument that he could not know about defendant's insanity internally invalid by moving an undercutter.

$\alpha_{25}\left[\pi_{22}\right]: \pi_{22}$ is not conclusive since check register

Then the adjudicator terminates the decision phase, thus implicitly ruling all remaining arguments internally valid by moving no undercutters and ruling them dialectically valid by moving no other counterarguments.

$\alpha_{26}$ : terminate

Defendant is the current winner so the adjudicator has in fact ruled that defendant wins the dispute. 
Figures 1, 2 and 3 display, respectively, the dialogue in reply form (in two parts) and the dialectical graph created during the dialogue. In Figures 1 and 2 a solid box means that a move is in and a dotted box that it is out in the dialogue game. As explained in Section 3.3 above, the dialectical graph records all arguments that were stepwise constructed during the dialogue, as well as their defeat relations. In Figure 3 a solid box means that the argument is justified and a dotted box that it is overruled relative to the set of all arguments contained in the graph.

The dialectical graph in fact contains a winning strategy for plaintiff in the embedded argument game on the basis of the statements made during the dialogue. Against both of defendant's attacks on his main argument he has a winning counterattack and defendant cannot construct other counterarguments without introducing new premises. At first sight, this would seem to be at odds with the outcome of the dialogue. However, plaintiff's argument $\pi_{18}$ was ruled illegal by the adjudicator so its premises must be disregarded in verifying correspondence. The remaining graph instead displays a winning strategy for defendant on the basis of all defended statements made during the dialogue, which agrees with the outcome of the dialogue.

Let us finally briefly illustrate distributions of the burden of persuasion over the adversaries. Assume that defendant has the burden of persuasion for her claim that there is something wrong with the signatures of the contract $\left(\delta_{12}\right)$. In that case, plaintiff can repeat his argument $\pi_{11}$ in attack on $\delta_{12}$, since in the embedded argument game the non-repetition rule now holds for defendant as regards ' $\neg$ signed'. Assume the dialogue then proceeds as above with $\delta_{13}$. If the adjudicator still assesses the argument for and against 'signed' as he did above, then in the decision phase he does not have to move a priority argument against $\delta_{12}$, since plaintiff is already winning this line of dispute.

\section{Conclusion}

The main purpose of this paper was to show how current dialogical models of legal procedure and persuasion dialogue can be extended to models of adjudication dialogue with a realistic role of the adjudicator. To this end, a formal model has been developed of an artificial but fairly typical adjudication procedure, using tools from argumentation theory (dialogue games) and artificial intelligence (logics for defeasible argumentation). The main feature of the model is a division into a pleadings phase, where the adversaries plea their case and the adjudicator has a largely mediating role, and a decision phase, where the adjudicator decides the dispute on the basis of the things put forward in the pleadings phase.

The model allows for decisions on legal-procedural correctness of dialogue utterances in the pleadings phase, such as on admissibility of evidence. The model also respects the legal distinction between the burdens of production and persuasion. It allows for explicit decisions on the burden of production in the pleadings phase and leaves room for verifying the burden of persuasion in the decision phase. The model also captures the notion of tactical burden of proof, by way of the notion of dialogical status of moves. If an adversary succeeds in changing the dialogical status of the main claim or argument his or her way, then s/he thereby shifts the tactical burden on the other player to change that status. Unlike the burdens of production and persuasion, which are imposed by law or the adjudicator, the tactical burden is automatically induced by the defeasible nature of the underlying logic and the dialectical nature of the dialogue game. Thus the model reconciles logical aspects of burden of proof induced by the defeasible nature of arguments with dialogical aspects of burden of proof as something that can be allocated by explicit decisions on legal grounds.

In future research it would be interesting to verify whether formal correspondence results can be proven similar to those for the two-player setup of Prakken (2005) (see Section 3.3 above). Also, the present dialogue game can be extended in various ways. For instance, debates on move legality could be modelled and the dialogue game could be combined with recent attempts to formalise debates about the burdens of production (Prakken et al.; 2005) and persuasion (Prakken and Sartor; 2007).

The reader may wonder what is the point of developing formal models of adjudication pro- 


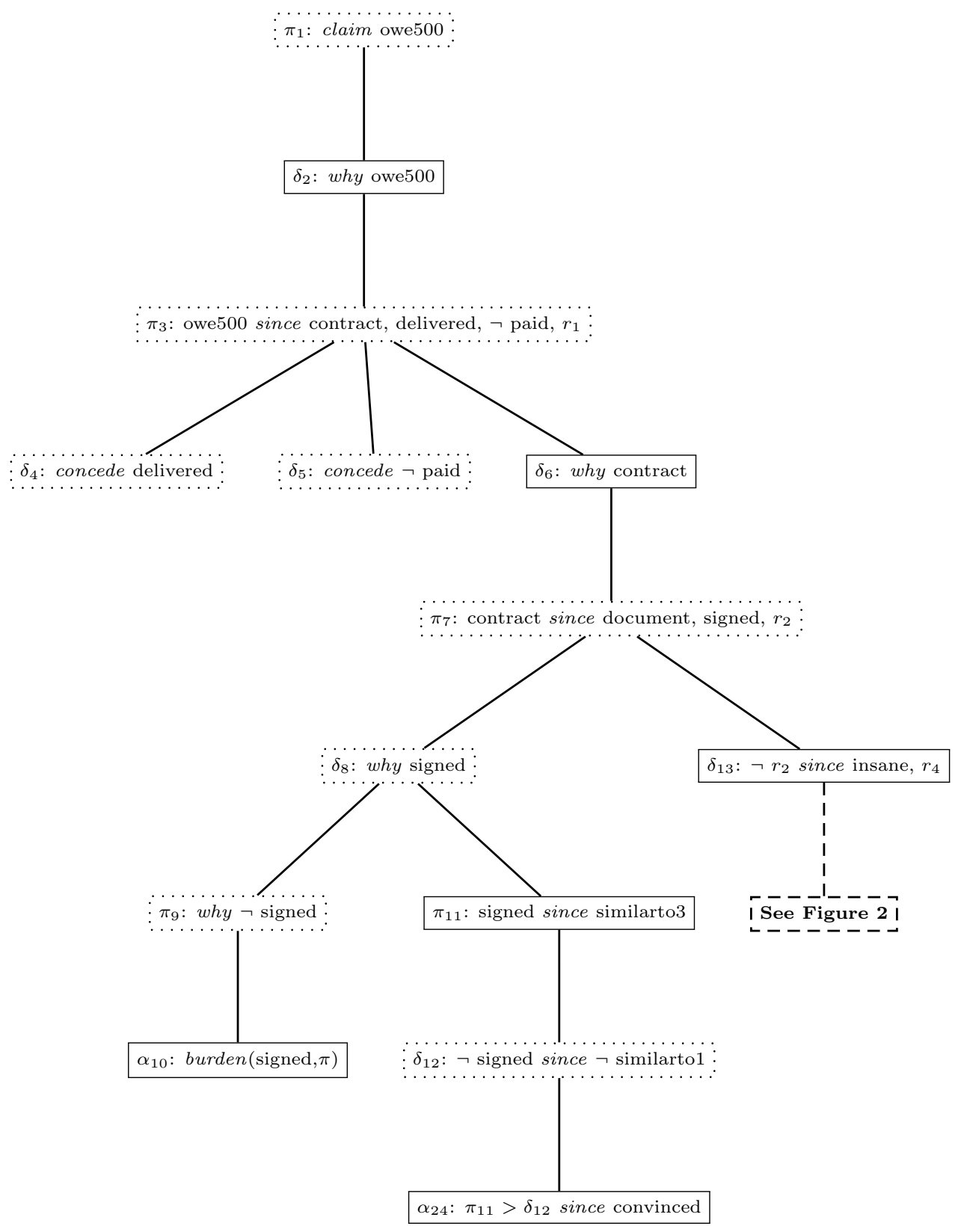

Figure 1: A dialogue tree (part 1) 


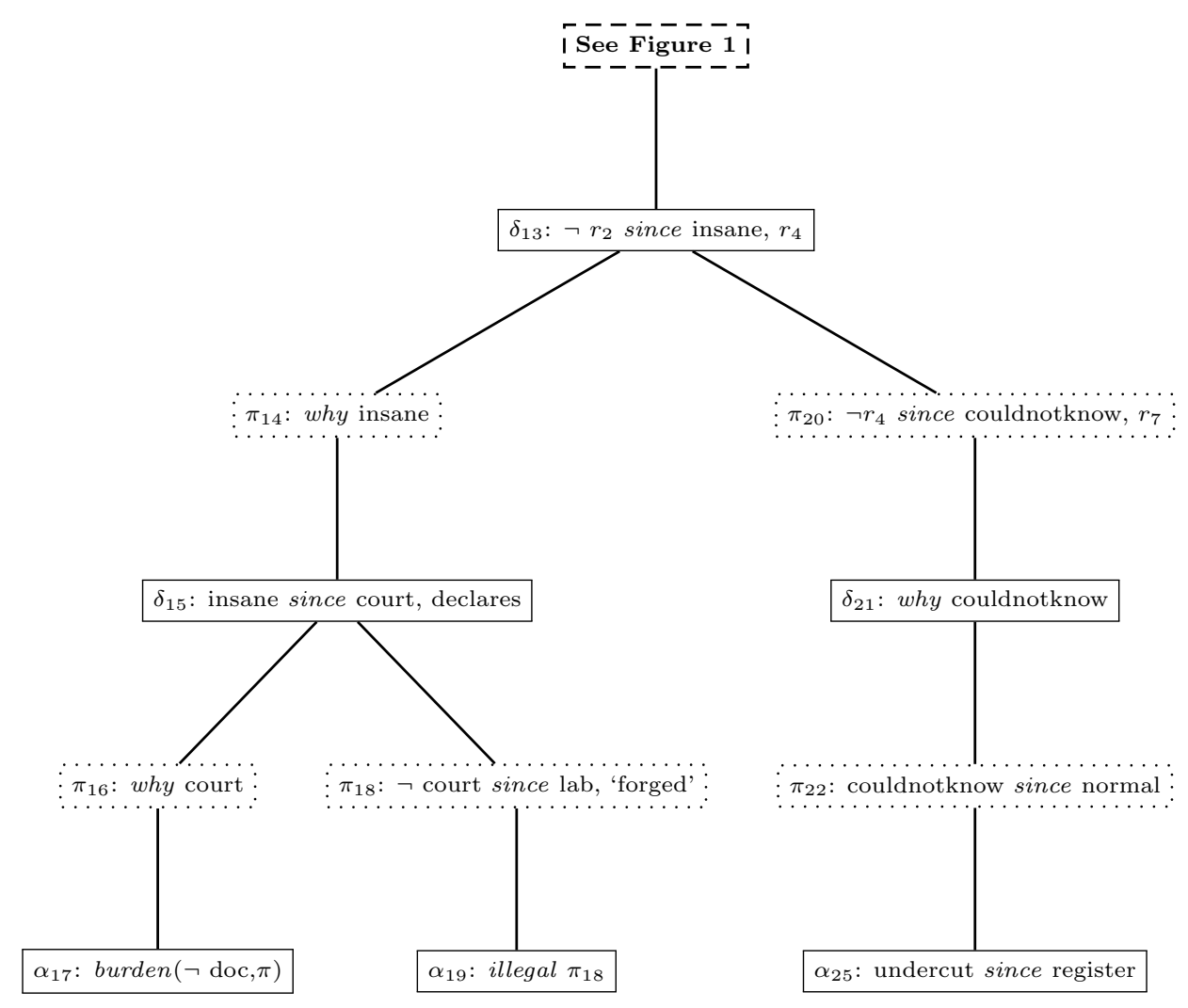

Figure 2: A dialogue tree (part 2) 


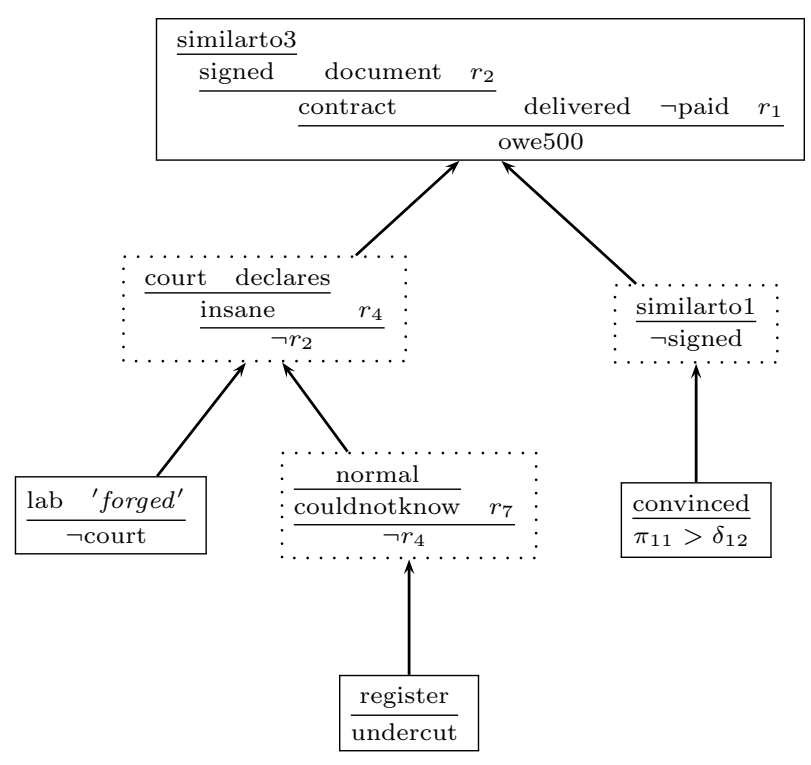

Figure 3: A dialectical graph

cedures. As said above in Section 3.1, the main aim of dialogue systems is to define and study conventions for coherent discourse, where in adjudication dialogues coherence can be defined as contributing to fair and effective dispute resolution. The point of this is partly analytical: by formalising and studying artificial procedures, insight can be obtained in their properties, in their relation with logic, in how differences in procedures can be understood as variations on certain elements, and so on. But this also has a normative point. Desirable properties can be formulated, i.e., properties which make procedures fair and/or effective, and procedures can be tested on whether they have these properties. Also, the rules of a specific procedure can be used to assess the quality of an adjudicator's decision from this point of view.

\section{Acknowledgement}

This research was partially supported by the EU under IST-FP6-002307 (ASPIC). I thank Burkhard Schäfer, Chris Reed, Giovanni Sartor and Doug Walton for useful discussions on the various aspects of burden of proof.

\section{References}

Bench-Capon, T. (1998). Specification and implementation of Toulmin dialogue game, Legal Knowledge-Based Systems. JURIX: The Eleventh Conference, Gerard Noodt Instituut, Nijmegen, pp. 5-19.

Bench-Capon, T., Geldard, T. and Leng, P. (2000). A method for the computational modelling of dialectical argument with dialogue games, Artificial Intelligence and Law 8: 233-254.

Brewka, G. (2001). Dynamic argument systems: a formal model of argumentation processes based on situation calculus, Journal of Logic and Computation 11: 257-282.

Carlson, L. (1983). Dialogue Games: an Approach to Discourse Analysis, Reidel Publishing Company, Dordrecht.

Gordon, T. (1993). The Pleadings Game: formalizing procedural justice, Proceedings of the Fourth International Conference on Artificial Intelligence and Law, ACM Press, New York, pp. 10-19. 
Hage, J. (1997). Reasoning With Rules. An Essay on Legal Reasoning and Its Underlying Logic, Law and Philosophy Library, Kluwer Academic Publishers, Dordrecht/Boston/London.

Hage, J. (2000). Dialectical models in artificial intelligence and law, Artificial Intelligence and Law 8: 137-172.

Hage, J., Leenes, R. and Lodder, A. (1994). Hard cases: a procedural approach, Artificial Intelligence and Law 2: 113-166.

Hamblin, C. (1971). Mathematical models of dialogue, Theoria 37: 130-155.

Leenes, R. (2001). Burden of proof in dialogue games and Dutch civil procedure, Proceedings of the Eighth International Conference on Artificial Intelligence and Law, ACM Press, New York, pp. 109-118.

Lodder, A. (1999). DiaLaw. On Legal Justification and Dialogical Models of Argumentation, Law and Philosophy Library, Kluwer Academic Publishers, Dordrecht/Boston/London.

Loui, R. (1998). Process and policy: resource-bounded non-demonstrative reasoning, Computational Intelligence 14: 1-38.

Mackenzie, J. (1979). Question-begging in non-cumulative systems, Journal of Philosophical Logic 8: $117-133$.

Peczenik, A. (1996). Jumps and logic in the law, Artificial Intelligence and Law 4: 297-329.

Prakken, H. (2001a). Modelling defeasibility in law: logic or procedure?, Fundamenta Informaticae 48: $253-271$.

Prakken, H. (2001b). Modelling reasoning about evidence in legal procedure, Proceedings of the Eighth International Conference on Artificial Intelligence and Law, ACM Press, New York, pp. 119-128.

Prakken, H. (2005). Coherence and flexibility in dialogue games for argumentation, Journal of Logic and Computation 15: 1009-1040.

Prakken, H. (2006). Formal systems for persuasion dialogue, The Knowledge Engineering Review 21: $163-188$.

Prakken, H. and Gordon, T. (1998). Rules of order for electronic group decision making - a formalization methodology, in J. Padget (ed.), Collaboration between human and artificial societies. Coordination and agent-based distributed computing, number 1624 in Springer Lecture Notes in AI, Springer Verlag, Berlin, pp. 246-263.

Prakken, H., Reed, C. and Walton, D. (2005). Dialogues about the burden of proof, Proceedings of the Tenth International Conference on Artificial Intelligence and Law, ACM Press, New York, pp. 115-124.

Prakken, H. and Sartor, G. (1997). Argument-based extended logic programming with defeasible priorities, Journal of Applied Non-classical Logics 7: 25-75.

Prakken, H. and Sartor, G. (2002). The role of logic in computational models of legal argument: a critical survey, in A. Kakas and F. Sadri (eds), Logic Programming and Beyond. Essays In Honour of Robert A. Kowalski, Part II, number 2048 in Springer Lecture Notes in Computer Science, Springer Verlag, Berlin, pp. 342-380.

Prakken, H. and Sartor, G. (2006). Presumptions and burdens of proof, in T. M. v. Engers (ed.), Legal Knowledge and Information Systems. JURIX 2006: The Nineteenth Annual Conference, IOS Press, Amsterdam etc., pp. 21-30. 
Prakken, H. and Sartor, G. (2007). Formalising arguments about the burden of persuasion, Proceedings of the Eleventh International Conference on Artificial Intelligence and Law, ACM Press, New York, pp. 97-106.

Prakken, H. and Vreeswijk, G. (2002). Logics for defeasible argumentation, in D. Gabbay and F. Günthner (eds), Handbook of Philosophical Logic, second edn, Vol. 4, Kluwer Academic Publishers, Dordrecht/Boston/London, pp. 219-318.

Rescher, N. (1977). Dialectics: a Controversy-oriented Approach to the Theory of Knowledge, State University of New York Press, Albany, N.Y.

Sartor, G. (1995). Defeasibility in legal reasoning, in Z. Bankowski, I. White and U. Hahn (eds), Informatics and the Foundations of Legal Reasoning, Law and Philosophy Library, Kluwer Academic Publishers, Dordrecht/Boston/London, pp. 119-157.

Sartor, G. (2005). Legal Reasoning: a Cognitive Approach to the Law, Springer Verlag, Berlin.

Strong, J. (ed.) (1992). McCormick on Evidence, 4 edn, West Publishing Co., St Paul.

Toulmin, S. (1958). The Uses of Argument, Cambridge University Press, Cambridge.

Vreeswijk, G. (2000). Representation of formal dispute with a standing order, Artificial Intelligence and Law 8: 205-231.

Walton, D. and Krabbe, E. (1995). Commitment in Dialogue. Basic Concepts of Interpersonal Reasoning, State University of New York Press, Albany, NY. 